\title{
Validation of reference genes for gene expression studies in tartary buckwheat (Fagopyrum tataricum Gaertn.) using quantitative real-time PCR
}

Chenglei Li ${ }^{1}$, Haixia Zhao ${ }^{1}$, Maofei Li ${ }^{1}$, Panfeng Yao ${ }^{1}$, Qingqing Li ${ }^{1}$, Xuerong Zhao ${ }^{1}$, Anhu Wang ${ }^{2}$, Hui Chen ${ }^{1}$, Zizhong Tang ${ }^{1}$, Tongliang Bu ${ }^{1}$, Qi Wu ${ }^{\text {Corresp. }}{ }^{1}$

${ }^{1}$ College of life science, Sichuan Agricultural University, Ya'an, Sichuan, China

2 Xichang College, Xichang, Sichuan, China

Corresponding Author: Qi Wu

Email address: wuqi@sicau.edu.cn

Quantitative real-time reverse transcriptase polymerase chain reaction ( $q R T-P C R$ ) is a sensitive technique for quantifying gene expression levels. By implementing three distinct algorithms (geNorm, normFinder and BestKeeper), we have validated the stability of the expression of seven candidate reference genes in tartary buckwheat, including FtSAND, FtCACS, FtExpressed1, FtGAPDH, FtActin, FtEF-1a and FtH3. In this study, the results indicated that FtCACS and FtSAND were the best reference genes for 'abiotic cotyledons', and FtExpressed 1 and FtEF-1 $\alpha$ were the best reference genes for aluminium treatment; FtCACS and FtExpressed 1 performed the best for the immature seed stage; FtCACS was best for the abiotic treatment, and FtH3 appeared to be the most suitable reference gene for the abiotic treatment in hypocotyls and all samples in this study. In contrast, FtActin and FtGAPDH are unsuitable genes. Our findings offer additional stable reference genes for gene expression research on tartary buckwheat at the immature seed stage and under abiotic treatment. 
1 Validation of reference genes for gene expression studies

2 in tartary buckwheat (Fagopyrum tataricum Gaertn.) using

3

4

5 Chenglei $\mathrm{Li}^{1}$, Haixia Zhao ${ }^{1}$, Maofei $\mathrm{Li}^{1}$, Panfeng $\mathrm{Yao}^{1}$, Qingqing $\mathrm{Li}^{1}$, Xuerong Zhao ${ }^{1}$, Anhu

6 Wang $^{2}$, Hui Chen ${ }^{1}$, Zizhong Tang ${ }^{1}$, Tongliang $\mathrm{Bu}^{1}$, Qi Wu${ }^{1 *}$

7

8

9

10

11

12

13

14

15

16

17

18

19

20

21

22

23

24

25

26

27

\section{quantitative real-time PCR}

${ }^{1}$ College of life science, Sichuan Agricultural university, Ya'an 625014, Sichuan, China

${ }^{2}$ Xichang college, Xichang 615013, Sichuan, China

*Corresponding Author: Qi Wu

Sichuan Agricultural university, Ya'an 625014, Sichuan, China

Email addresses: wuqi@sicau.edu.cn

\section{Abstract}

Quantitative real-time reverse transcriptase polymerase chain reaction (qRT-PCR) is a sensitive technique for quantifying gene expression levels. By implementing three distinct algorithms (geNorm, normFinder and BestKeeper), we have validated the stability of the expression of seven candidate reference genes in tartary buckwheat, including FtSAND, FtCACS, FtExpressed1, FtGAPDH, FtActin, FtEF-1a and FtH3. In this study, the results indicated that FtCACS and FtSAND were the best reference genes for 'abiotic cotyledons', and FtExpressedl and FtEF-1 $\alpha$ were the best reference genes for aluminium treatment; FtCACS and FtExpressed 1 performed the best for the immature seed stage; FtCACS was best for the abiotic treatment, and FtH3 appeared to be the most suitable reference gene for the abiotic treatment in hypocotyls and all samples in this study. In contrast, FtActin and FtGAPDH are unsuitable genes. Our findings offer additional stable reference genes for gene expression research on tartary buckwheat at the immature seed stage and under abiotic treatment. 


\section{Introduction}

29 Quantitative real-time reverse transcription polymerase chain reaction (qRT-PCR) has become 30 the most prevalent quantification method used in assays of gene expression on account of its specificity, accuracy, efficiency and high sensitivity (Jiang et al. 2014). The data from qRT-PCR can be analysed using absolute or relative quantification. Absolute quantification yields an exact gene copy number by transforming quantification cycles (Hruz et al.) into a standard curve. Relative quantification relies on internal control genes as references to present the qRT-PCR data of the target genes (Ginzinger 2002). Relative quantification has become a more widely used method in gene expression assays because most researchers mainly focus on the analysis of differences in gene expression ( $\mathrm{Wu}$ et al. 2016). To normalize qRT-PCR data, a reference gene is needed, and it should be universally valid, with a similar expression level across all feasible cells, tissue samples and experimental treatments (Huang et al. 2014). However, ideal reference genes do not actually exist (Remans et al. 2014). The selection of the appropriate reference gene is a critical step in controlling the variability of samples when using a sensitive qRT-PCR technique (Tong et al. 2009). If the reference gene is appropriate, the discrepancies that may exist in terms of the initial sample amount, RNA integrity, RNA recovery, and the efficiency of cDNA synthesis will be eliminated. Statistical algorithms, such as geNorm (Vandesompele et al. 2002), normFinder (Andersen et al. 2004) and BestKeeper (Pfaffl et al. 2004), have been developed to help to select appropriate reference genes.

Most studies about gene expression in bacteria (Wu et al. 2017), yeast (Cankorur-Cetinkaya et al. 2012) and mammals (Terzi et al. 2010) now include reference gene validation (Chapman \& Waldenstrom 2015). However, the validation of reference genes in plants has received very little attention, and housekeeping genes tend to be used as references without any appropriate validation (Gutierrez et al. 2008). In 2005, Czechowski et al were the first to present a list of stably expressed Arabidopsis genes under a large range of experimental conditions, and the evidence clearly showed that several genes are expressed more stably than traditional reference genes (Czechowski et al. 2005). Since then, suitable reference genes for gene expression studies have been reported in higher plants such as flax (Huis et al. 2010), soybean (Hu et al. 2009), tomato (Lovdal \& Lillo 2009), Pisum sativum (Die et al. 2010), Brachypodium distachyon (Hong et al. 2008) and carrot (Tian et al. 2015). However, reference gene stability is not consistent 
58

59

60

61

62

63

64

65

66

67

68

69

70

71

72

73

74

75

76

77

78

79

80

81

82

83

84

85

86

87

88

across experimental conditions and plant species. Consequently, it is necessary to find additional reference genes for different conditions and species.

Tartary buckwheat (Fagopyrum tataricum Gaertn.) belongs to the Polygonaceae family (Kim et al. 2013), and as an important functional food material, it has a relatively high flavonoid content. Tartary buckwheat is capable of thriving in regions with poor soil or harsh climates (Kim et al. 2009). The results of genome sequencing also show that tartary buckwheat has a remarkable ability to cope with highly variable environmental stress, including drought, salinity, UV-B and cold (Zhang et al. 2017). The stress resistance of tartary buckwheat is mainly due to its abundant flavonoids (Suzuki et al. 2005). In particular, tartary buckwheat is a naturally aluminium (Al) tolerant species (Wang et al. 2015). Abiotic stresses and flavonoid metabolism regulate the expression of these genes in plants at both transcriptional and post-transcriptional levels. There are many reports on tartary buckwheat under various experimental conditions in which gene expression is normalized to a reference gene $(H 3)$ for semi-quantitative RT-PCR or qRT-PCR (Bai et al. 2014). However, to date, there is no systematic strategy to analyse tartary buckwheat reference genes at the immature seed stage or under abiotic stress.

In this work, we aimed to evaluate the potential use of different reference genes for internal normalization to more accurately measure the expression level of genes of interest in tartary buckwheat. Seven candidate reference genes were selected, and the stability of their expression was assessed in tartary buckwheat at the immature seed stage and under different abiotic stress treatments. Evaluating the stability of the expression of candidate reference genes depends on statistical analysis. Three different statistical software programs (geNorm, normFinder, and BestKeeper) were used to calculate the variability of the expression of the candidate genes and determine which were the most suitable.

\section{Materials \& Methods}

\section{Plant materials and treatments}

Tartary buckwheat (“Xiqiao No. 2") seeds were grown in the field on a farm (XTBG; 2959'N, 102 59'E; 800 m elevation) at Sichuan Agricultural University, Ya’an, Sichuan, China (Li et al. 2012). Tissues, including roots, stems, leaves, flowers, immature seed 1 (seed formation started) and immature seed 2 (seeds in the milk) were collected at the immature seed stage (Gupta et al. 2011). The 7-day-old seedlings were stressed with saline or drought by adding $100 \mathrm{mM} \mathrm{NaCl}$ or 
89

90

91

92

93

94

95

96

97

98

99

100

101

102

103

104

105

106

107

108

109

110

111

112

113

114

115

116

117

118

119

20\% PEG 2000, respectively, to the medium. The 7-day-old seedlings were exposed in a chamber at $4^{\circ} \mathrm{C}$ with a $16 \mathrm{~h}$ photoperiod for cold treatment (Gao et al. 2016). UV-B treatment was conducted under UV-B $\left(302 \mathrm{~nm}, 0.1 \mathrm{~mW} / \mathrm{cm}^{2}\right)$ in a chamber . After 0, 2, 4, 6, 12 and $24 \mathrm{~h}$ of treatment, all stressed seedlings were collected and separated into cotyledons and hypocotyls. For the $\mathrm{Al}$ treatment, the samples were processed according to a previous report (Zhu et al. 2015). Root tips $(0-2 \mathrm{~cm})$ and basal roots $(2-4 \mathrm{~cm})$ were sampled under both $-\mathrm{Al}$ and $+\mathrm{Al}$ conditions. All samples were collected in two biological replicates, and RNA was extracted immediately.

\section{Total RNA isolation and cDNA synthesis}

Total RNA was isolated from various samples with an RNAout 2.0 kit (Tiandz, China) according to the manufacturer's instructions. To remove trace DNA from samples, total RNA extractions were treated with RNase free DNase I. The RNA integrity was detected using $2 \%$ agarose gels. A Bio-RAD smart $\operatorname{spec}^{\mathrm{TM}}$ plus spectrophotometer was used to determine the RNA purity. cDNA was synthesized with a PrimeScript ${ }^{\mathrm{TM}} \mathrm{RT}$ reagent kit and gDNA Eraser (Perfect Real Time) (TaKaRa, Dalian, China).

\section{Selection of candidate reference genes and design of qRT-PCR primers}

Potential homologues of the seven candidate genes were identified from the transcriptome sequencing data of tartary buckwheat ('Xiqiao No. 2') (Yao et al. 2017). Primers were designed using Primer Premier 5.0. All primers used in this research are listed in Table 1. The specificity of the amplification was assessed based on the presence of a single band of the expected size in a $1.5 \%$ agarose gel following electrophoresis and a single peak in the qRT-PCR melting curve.

\section{Quantitative real-time PCR}

The qRT-PCR procedure was designed in accordance with MIQE guidelines (Bustin et al. 2009). qRT-PCR was executed in a CFX96 Real Time PCR system (Bio Rad, Singapore) with a SYBR Premix EX Taq kit (TaKaRa, Japan) in a total reaction volume of $15 \mu \mathrm{L}$ that included 10 $\mu \mathrm{L}$ of SYBR Green mix, primers at $0.5 \mu \mathrm{M}$ each and $1 \mu \mathrm{L}$ of cDNA. The amplification conditions were as follows: $95^{\circ} \mathrm{C}$ for $30 \mathrm{~s}$ and 40 cycles of $95^{\circ} \mathrm{C}$ for $5 \mathrm{~s}$ and $60^{\circ} \mathrm{C}$ for $20 \mathrm{~s}$. A melting curve from 60 to $95^{\circ} \mathrm{C}$ was used to verify the specificity of the PCR amplification. All genes were amplified from cDNA. Ten-fold serial dilutions of cDNA samples from young leaves were used to establish the standard curves to calculate the amplification efficiency of each primer pair. 


\section{Statistical analysis}

121 Three software programs (geNorm v3.5, the Excel add in of normFinder v0.953 and 122 BestKeeper v1) were used to analysis the stability of reference gene expression across all 123 experimental sets (Andersen et al. 2004; Pfaffl et al. 2004; Vandesompele et al. 2002). SPSS 124 v17.0 was used to calculate the span of $\mathrm{Cq}$ values for each gene by drawing a box-whisker plot, 125 and the expression levels of FtSTAR and FtDFR were showed by mean \pm standard deviation 126 (SD).

127

128

\section{Results}

\section{RNA solution and quality}

A series of 58 samples from tartary buckwheat were divided into six different groups. 'Abiotic cotyledons' was composed of cotyledons from all stress-treated samples. 'Abiotic hypocotyls' included hypocotyls from all stress-treated samples. 'Abiotic total' was composed of 'abiotic cotyledons' and 'abiotic hypocotyls'. 'Al treatment' comprised all samples that were treated with Al. 'Immature seed stage' consisted of six tissues (roots, stems, leaves, flowers, immature seed 1 included all the samples in this study. Protein and organic pollutants were isolated and removed from all samples via RNA extraction. The total RNAs, with $A_{260} / A_{280}$ ratios of $1.8-2.0$, were reverse transcribed into cDNA as templates for qRT-PCR detection.

\section{Expression profiles of candidate reference genes}

We selected the best reference genes (among the 7 candidate genes) from the seven candidate genes in six different groups (sequencing data was shown in Supplemental Fig. S1). The amplification efficiencies (E) of all reactions ranged from $95.5 \%$ to $107.5 \%$ and were calculated from standard curves with good linear relationships $\left(R^{2}>0.99\right)$. Single-peak melting curves were obtained for all qRT-PCR amplifications (Supplemental Fig. S2). A simple and commonly used method to identify stably expressed genes is to compare the span of quantification cycle (Hruz et al.) values for each gene in the qRT-PCR reactions. The results showed that the candidate reference genes spanned a wide range of $\mathrm{Cq}$ values, ranging from 14.98 (FtGAPDH) to 29.65

148 (FtActin), with the median $\mathrm{Cq}$ values of the genes ranging from 17.59 (FtGADPH) to 25.5 149 (FtSAND). The reference gene expression levels, in descending order, were FtGAPDH, FtEF-1, 150 FtH3, FtCACS, FtActin, FtExpressed1 and FtSAND. FtActin (median, 23.55; Cq variation, 
151 11.38) showed the most variability, and the FtH3 gene (median, 20.19; Cq variation, 3.67) 152 showed the least variability (Fig. 1).

153 Software: geNorm, NormFinder and BestKeeper

154 After a simple comparison of the raw $\mathrm{Cq}$ values, the three software programs were used to 155 further analyse the stability of the expression of the seven candidate genes. The genes were 156 ranked in descending order in terms of the stability of their expression in each of six groups

157 (Table 2), and the details of the cotyledons and hypocotyls under abiotic stress are provided in 158 Supplemental Table S1. GeNorm performed a stepwise exclusion of the most unstable gene and 159 then recalculated $M$ until only two genes remained, and these two genes had the most stable 160 expression (Vandesompele et al. 2002). The gene with the lower M value was considered to have 161 the most stable expression. An $M$ value limit of $<1.5$ was suggested by geNorm. All the results 162 from geNorm were lower than 1.5 in 'abiotic cotyledons' and 'total'; FtCACS and FtH3 (M = $1630.33,0.60)$ were chosen as the most stable genes, while FtH3 and FtSAND $(\mathrm{M}=0.65,0.55)$ were 164 identified as the most stable genes in the 'abiotic hypocotyls' and 'abiotic total' groups, 165 respectively, In 'immature seed stage', FtCACS and FtExpressed1 $(\mathrm{M}=0.16)$ were recognized 166 as the most stable genes. Finally, FtEF-1 $\alpha$ and FtExpressed $1(\mathrm{M}=0.16)$ were the most stable 167 genes in 'Al treatment'.

168 To determine the optimal number of reference genes required for accurate normalization, 169 geNorm was used to calculate the pairwise variation $(\mathrm{Vn} / \mathrm{Vn}+1)$ between the sequential normalization factors (NFs) (NFn and NFn+1). As suggested, a threshold value of 0.15 was adopted. As depicted in Fig. 2, pairwise variation analysis indicated that the ideal number of reference genes may be different for the different groups. For instance, only two genes are necessary for normalization for 'immature seed stage', 'abiotic cotyledons' and 'Al treatment', but the pairwise variation of the other three groups was above the threshold of 0.15 . The pairwise variations for cotyledons and hypocotyls under different abiotic treatments are provided in Supplemental Fig. S3.

177 Unlike geNorm, normFinder (Andersen et al. 2004) depends on a variance estimation approach, which allows the comparison of inter/intra-group variation. Genes with the lowest average expression stability values are the most stable. In the normFinder analysis, FtH3 and FtCACS were the most stable genes in 'abiotic cotyledons', and FtSAND and FtExpressedl were the most stable genes under 'Al treatment'. In addition, the most stable reference genes in 
182 'immature seed stage' were FtSAND and FtGAPDH, while the other three groups had the same 183 two most stable reference genes (FtSAND and FtCACS). The least stable gene in all groups was 184 FtActin.

185 BestKeeper (Pfaffl et al. 2004) determines the most stable genes by taking the coefficient of 186 variance $(\mathrm{CV})$ and $\mathrm{SD}$ of the $\mathrm{Cq}$ values. The more stably expressed genes are indicated by the 187 lower SD and CV values. The results showed that the most stably expressed genes were FtSAND $188(\mathrm{CV} \pm \mathrm{SD}=1.32 \pm 0.33)$ and FtCACS $(\mathrm{CV} \pm \mathrm{SD}=1.47 \pm 0.31)$ for 'abiotic cotyledons'. In 189 'abiotic hypocotyls', FtH3 (CV $\pm \mathrm{SD}=2.47 \pm 0.51)$ and FtSAND $(\mathrm{CV} \pm \mathrm{SD}=2.68 \pm 0.69)$ were 190 the most stable genes. FtH3 $(\mathrm{CV} \pm \mathrm{SD}=2.55 \pm 0.52, \mathrm{CV} \pm \mathrm{SD}=2.69 \pm 0.54)$ and FtCACS (CV $191 \pm \mathrm{SD}=2.70 \pm 0.61, \mathrm{CV} \pm \mathrm{SD}=2.33 \pm 0.53$ ) were the most stable genes in the 'abiotic total' and 192 'total' groups, respectively. FtExpressed $1(\mathrm{CV} \pm \mathrm{SD}=073 \pm 0.17)$ and FtEF-1 $\alpha(\mathrm{CV} \pm \mathrm{SD}=$ $1931.55 \pm 0.25)$ showed the most stable expression in 'Al treatment', and FtExpressedl $(\mathrm{CV} \pm \mathrm{SD}=$ $1942.00 \pm 0.50)$ and FtCACS $(\mathrm{CV} \pm \mathrm{SD}=2.43 \pm 0.57)$ showed the highest expression stabilities in 195 'immature seed stage'. The least stable gene was FtActin, but the FtGAPDH gene, with a CV \pm 196 SD of $7.59 \pm 1.39$ in 'abiotic hypocotyls', was considered the least acceptable for gene 197 expression normalization.

\section{Reference gene validation}

199 To validate the availability of a reference gene, the expression levels of FtSTAR under Al 200 treatment and of the FtDFR gene under UV treatment were determined using the seven candidate 201 reference genes for normalization. For Al treatment, STAR has a conserved response in plants, 202 and this has been shown in previous reports, such as reports on rice STAR2 (Huang et al. 2009), 203 Arabidopsis ALS (Larsen et al. 2005) and tartary buckwheat FtSTAR2 (Zhu et al. 2015), whose 204 expression increased after exposure to Al. The expression of FtSTAR2 was greatly increased 205 (Fig. 3), which was reinforced by the Al-induced expression of STAR2. The use of the most 206 favourable reference gene (FtExpressed1) resulted in the greatest variation, resulting in increases 207 of approximately 1.67-fold in root tips and 6.24-fold in basal roots, and the use of the other most 208 stable gene $(F t E F-1 a)$ resulted in increases of approximately 1.42-fold and 6.19-fold, 209 respectively. Finally, the use of the least stable gene (FtActin) resulted in increases of 210 approximately 1.90-fold in root tips and 2.48-fold in basal roots. These genes also showed a 211 pattern of increased expression, regardless of whether stable or unstable reference genes were 212 used for normalization. However, the relative expression using the most stable genes showed the 
213 higher fold increases compared with the unstable genes. Therefore, FtExpressed 1 and FtEF-1a 214 are the most suitable genes under Al treatment.

215 DFR catalyses the reduction of dihydroflavonols to leuco-anthocyanins and is a key enzyme in 216 the biosynthesis of anthocyanins (Yuan et al. 2007). The contents of anthocyanin increased under 217 UV treatment in tartary buckwheat hypocotyls (Eguchi \& Sato 2009). In hypocotyls under UV 218 treatment, the relative quantification of FtDFR using the most stable genes (FtCACS, FtH3 and 219 FtActin) for normalization exhibited similar expression patterns but different expression levels. 220 When other less stable genes were used for normalization, the results obviously differed from 221 those with the most stable genes (Fig. 4). The expression levels of the FtDFR gene under UV 222 treatment showed similar trends after normalization to stable genes in hypocotyls. Thus, we 223 selected the most stable reference genes for gene expression normalization in tartary buckwheat.

224

\section{Discussion}

226 SYBR Green I is a fluorescent reporter dye used in qRT-PCR; when it binds double-stranded 227 DNA, its fluorescence increases nearly 1000-fold (Morrison et al. 1998). The problems in qRT228 PCR caused by the variability of RNA templates and inappropriate data normalization are 229 obvious and widely known but disregarded (Nolan et al. 2006). To address these questions, 230 proper reference genes should be used. Thus, validating candidate reference genes and selecting stable reference genes are becoming general interests for researchers.

Candidate reference genes can be selected from reference gene validation papers that use the 233 same experimental conditions or closely related species (orthologies) or by mining transcriptomic data for stably expressed genes or traditional housekeeping genes (Hruz et al. 235 2011). The seven candidate reference genes tested in tartary buckwheat include three genes that are stably expressed in common buckwheat (SAND, CACS and Expressed1), three commonly used reference genes (GAPDH, Actin and EF-1a) and one unique gene (H3). Neither high (>30) nor low $(<15) \mathrm{Cq}$ values are recommended based on general guidelines (Wan et al. 2010). In this study, the Cq values of the reference genes ranged from 14.98 (FtGAPDH) to 29.65 (FtActin), suggesting variable expression. Although the raw $\mathrm{Cq}$ value comparison can provide a rough estimate of the stability of gene expression, it is not sufficient to accurately evaluate the expression patterns of reference genes. 
243 Scientists have developed several approaches to select stable reference genes for 244 normalization. However, to date, there is no consistent algorithm that can be used to test gene 245 stability. These software programs have their own merits and drawbacks: NormFinder can avoid 246 the misinterpretations caused by the artificial selection of co-regulated genes (Andersen et al. 247 2004); geNorm is based on the assumption that none of the analysed genes are co-regulated 248 (Vandesompele et al. 2002); and geNorm can estimate the fewest number of reference genes 249 needed for accurate normalization (Stamova et al. 2009). It seems possible that more reliable 250 controls can be obtained using a combination of different algorithms (de Almeida et al. 2010). 251 We used three software programs that were based on different statistical approaches to assess 252 seven candidate genes in tartary buckwheat. The distinct statistical algorithms are likely to 253 generate inconsistent rankings of stability. After integrating the outcomes of the three programs 254 above in this study, we recommend the reference genes in tartary buckwheat as follows: FtCACS $255+F t S A N D$ for 'abiotic cotyledons'; FtExpressed1 + FtEF-1 $\alpha$ for 'Al treatment'; FtCACS + 256 FtExpressed1 for 'immature seed stage'; FtCACS for 'abiotic total'; and FtH3 for 'abiotic 257 hypocotyls' and 'total'. The $H 3$ gene is the most commonly used internal gene for normalization 258 in tartary buckwheat (Luo et al. 2016). Our results indicate that FtH3 is a stable gene under 259 abiotic treatment. However, it is not stable across different organs or after Al treatment. In 260 common buckwheat (Demidenko et al. 2011), CACS and Expressedl were validated as the most 261 stable genes in the development and fruit stages. FtCACS and FtExpressedl being the most 262 stable reference genes in the immature seed stage of tartary buckwheat supports the statement 263 that the orthologues of identified reference genes could serve the same purpose in other species. 264 In tomato, similar results were obtained in a study on reference gene selection (Exposito265 Rodriguez et al. 2008).

266 GAPDH and Actin are the most generally used reference genes for the analysis of gene 267 expression in various plant species (Kumar et al. 2011). However, our analysis indicates that $268 G A P D H$ and Actin are not reliable genes for comparative expression analysis. The cause of these 269 fluctuations at the gene expression level is probably that GAPDH and Actin have several 270 biological functions, such as participating in the glycolytic pathway and other processes 271 (Stürzenbaum \& Kille 2001). Actin supports the cell and determines its shape, and it also takes 272 part in other cellular functions (Kravets et al. 2017). Although Actin is a stable reference gene in 273 developmental studies, it is not stable under various conditions. 
274

275

276

277

278

279

280

281

282

283

284

285

286

287

288

289

290

291

292

293

294

295

296

297

298

299

300

301

302

303

304

305

306

307

308

309

310

311

312

313

314

315

316

317

\section{Conclusions}

As far as we know, our studies take advantage of three software (geNorm, NormFinder and BestKeeper) to analysis the stability of seven candidate reference genes for the first time in tartary buckwheat using qRT-PCR. Three software identified sightly differet genes as most suited for normalization prompted us to merge the data. The result showed that the expression of $G A P D H$ or Actin is unstable across all samples. We also provide a list with the stable reference genes in six group and under certain conditions.

\section{References}

Andersen CL, Jensen JL, and Orntoft TF. 2004. Normalization of real-time quantitative reverse transcription-PCR data: a model-based variance estimation approach to identify genes suited for normalization, applied to bladder and colon cancer data sets. Cancer Res 64:5245-5250. 10.1158/0008-5472.CAN-04-0496

Bai YC, Li CL, Zhang JW, Li SJ, Luo XP, Yao HP, Chen H, Zhao HX, Park SU, and Wu Q. 2014. Characterization of two tartary buckwheat R2R3-MYB transcription factors and their regulation of proanthocyanidin biosynthesis. Physiol Plant 152:431-440. 10.1111/ppl.12199

Bustin SA, Benes V, Garson JA, Hellemans J, Huggett J, Kubista M, Mueller R, Nolan T, Pfaffl MW, Shipley GL, Vandesompele J, and Wittwer CT. 2009. The MIQE guidelines: minimum information for publication of quantitative real-time PCR experiments. Clin Chem 55:611-622. 10.1373/clinchem.2008.112797

Cankorur-Cetinkaya A, Dereli E, Eraslan S, Karabekmez E, Dikicioglu D, and Kirdar B. 2012. A novel strategy for selection and validation of reference genes in dynamic multidimensional experimental design in yeast. PLoS One 7:e38351. 10.1371/journal.pone.0038351

Chapman JR, and Waldenstrom J. 2015. With Reference to Reference Genes: A Systematic Review of Endogenous Controls in Gene Expression Studies. PLoS One 10:e0141853. 10.1371/journal.pone.0141853

Czechowski T, Stitt M, Altmann T, Udvardi MK, and Scheible WR. 2005. Genome-wide identification and testing of superior reference genes for transcript normalization in Arabidopsis. Plant Physiol 139:5-17. 10.1104/pp.105.063743

de Almeida MR, Ruedell CM, Ricachenevsky FK, Sperotto RA, Pasquali G, and Fett-Neto AG. 2010. Reference gene selection for quantitative reverse transcriptionpolymerase chain reaction normalization during in vitro adventitious rooting in Eucalyptus globulus Labill. BMC Mol Biol 11:73. 10.1186/1471-2199-11-73

Demidenko NV, Logacheva MD, and Penin AA. 2011. Selection and validation of reference genes for quantitative real-time PCR in buckwheat (Fagopyrum esculentum) based on transcriptome sequence data. PLoS One 6:e19434. 10.1371/journal.pone.0019434

Die JV, Roman B, Nadal S, and Gonzalez-Verdejo Cl. 2010. Evaluation of candidate reference genes for expression studies in Pisum sativum under different experimental conditions. Planta 232:145-153. 10.1007/s00425-010-1158-1

Eguchi K, and Sato T. 2009. Differences in the Ratios of Cyanidin-3-O-glucoside and Cyanidin-3-O-rutinocide to Total Anthocyanin under UV and Non-UV Conditions in 
318

319

320

321

322

323

324

325

326

327

328

329

330

331

332

333

334

335

336

337

338

339

340

341

342

343

344

345

346

347

348

349

350

351

352

353

354

355

356

357

358

359

360

361

362

363

364

365

366

367

368

Tartary Buckwheat (Fagopyrum tataricum Garten). Plant Production Science 12:150-155. Doi 10.1626/Pps.12.150

Exposito-Rodriguez M, Borges AA, Borges-Perez A, and Perez JA. 2008. Selection of internal control genes for quantitative real-time RT-PCR studies during tomato development process. BMC Plant Biol 8:131. 10.1186/1471-2229-8-131

Gao F, Zhao HX, Yao HP, Li CL, Chen H, Wang AH, Park SU, and Wu Q. 2016. Identification, isolation and expression analysis of eight stress-related R2R3-MYB genes in tartary buckwheat (Fagopyrum tataricum). Plant Cell Rep 35:1385-1396. 10.1007/s00299-016-1971-5

Ginzinger DG. 2002. Gene quantification using real-time quantitative PCR. Experimental Hematology 30:503-512. 10.1016/s0301-472x(02)00806-8

Gupta N, Sharma SK, Rana JC, and Chauhan RS. 2011. Expression of flavonoid biosynthesis genes vis-a-vis rutin content variation in different growth stages of Fagopyrum species. Journal of Plant Physiology 168:2117-2123. 10.1016/j.jplph.2011.06.018

Gutierrez L, Mauriat M, Guenin S, Pelloux J, Lefebvre JF, Louvet R, Rusterucci C, Moritz T, Guerineau F, Bellini C, and Van Wuytswinkel O. 2008. The lack of a systematic validation of reference genes: a serious pitfall undervalued in reverse transcription-polymerase chain reaction (RT-PCR) analysis in plants. Plant Biotechnol J 6:609-618. 10.1111/j.1467-7652.2008.00346.x

Hong SY, Seo PJ, Yang MS, Xiang F, and Park CM. 2008. Exploring valid reference genes for gene expression studies in Brachypodium distachyon by real-time PCR. BMC Plant Biol 8:112. 10.1186/1471-2229-8-112

Hruz T, Wyss M, Docquier M, Pfaffl MW, Masanetz S, Borghi L, Verbrugghe P, Kalaydjieva L, Bleuler S, Laule O, Descombes P, Gruissem W, and Zimmermann P. 2011. RefGenes: identification of reliable and condition specific reference genes for RTqPCR data normalization. BMC Genomics 12:156. 10.1186/1471-2164-12-156

Hu R, Fan C, Li H, Zhang Q, and Fu YF. 2009. Evaluation of putative reference genes for gene expression normalization in soybean by quantitative real-time RT-PCR. BMC Mol Biol 10:93. 10.1186/1471-2199-10-93

Huang CF, Yamaji N, Mitani N, Yano M, Nagamura Y, and Ma JF. 2009. A bacterial-type $A B C$ transporter is involved in aluminum tolerance in rice. Plant Cell 21:655-667. 10.1105/tpc.108.064543

Huang L, Yan H, Jiang X, Yin G, Zhang X, Qi X, Zhang Y, Yan Y, Ma X, and Peng Y. 2014. Identification of candidate reference genes in perennial ryegrass for quantitative RT-PCR under various abiotic stress conditions. PLoS One 9:e93724. 10.1371/journal.pone.0093724

Huis R, Hawkins S, and Neutelings G. 2010. Selection of reference genes for quantitative gene expression normalization in flax (Linum usitatissimum L.). BMC Plant Biol 10:71. 10.1186/1471-2229-10-71

Jiang Q, Wang F, Li MY, Ma J, Tan GF, and Xiong AS. 2014. Selection of suitable reference genes for $\mathrm{qPCR}$ normalization under abiotic stresses in Oenanthe javanica (BI.) DC. PLoS One 9:e92262. 10.1371/journal.pone.0092262

Kim YB, Park SY, Thwe AA, Seo JM, Suzuki T, Kim SJ, Kim JK, and Park SU. 2013. Metabolomic analysis and differential expression of anthocyanin biosynthetic genes in white- and red-flowered buckwheat cultivars (Fagopyrum esculentum). $J$ Agric Food Chem 61:10525-10533. 10.1021/jf402258f

Kim YK, Li XH, Xu H, II Park N, Uddin MR, Pyon JY, and Park SU. 2009. Production of phenolic compounds in hairy root culture of tartary buckwheat (Fagopyrum tataricum Gaertn). Journal of Crop Science and Biotechnology 12:53-57. $10.1007 / \mathrm{s} 12892-009-0075-y$ 
369

370

371

372

373

374

375

376

377

378

379

380

381

382

383

384

385

386

387

388

389

390

391

392

393

394

395

396

397

398

399

400

401

402

403

404

405

406

407

408

409

410

411

412

413

414

415

416

417

418

419

Kravets EA, Yemets Al, and Blume YB. 2017. Cytoskeleton and nucleoskeleton involvement in processes of cytomixis in plants. Cell Biol Int. 10.1002/cbin.10842

Kumar V, Sharma R, Trivedi PC, Vyas GK, and Khandelwal V. 2011. Traditional and novel references towards systematic normalization of qRT-PCR data in plants. Australian Journal of Crop Science 5:1455-1468. 10.1016/j.fcr.2011.04.016

Larsen PB, Geisler MJ, Jones CA, Williams KM, and Cancel JD. 2005. ALS3 encodes a phloem-localized $A B C$ transporter-like protein that is required for aluminum tolerance in Arabidopsis. Plant J 41:353-363. 10.1111/j.1365-313X.2004.02306.x

Li C, Bai Y, Li S, Chen H, Han X, Zhao H, Shao J, Park SU, and Wu Q. 2012. Cloning, characterization, and activity analysis of a flavonol synthase gene FtFLS1 and its association with flavonoid content in tartary buckwheat. J Agric Food Chem 60:5161-5168. 10.1021/jf205192q

Lovdal T, and Lillo C. 2009. Reference gene selection for quantitative real-time PCR normalization in tomato subjected to nitrogen, cold, and light stress. Anal Biochem 387:238-242. 10.1016/j.ab.2009.01.024

Luo XP, Zhao HX, Xue J, Li CL, Chen H, Park SU, and Wu Q. 2016. Cloning of two basic helix-loop-helix transcription factor genes from Tartary buckwheat (Fagopyrum tataricum) and their expression under abiotic stress. Turkish Journal of Biology 40:1192-1201. 10.3906/biy-1511-36

Morrison TB, Weis JJ, and Wittwer CT. 1998. Quantification of low-copy transcripts by continuous SYBR Green I monitoring during amplification. Biotechniques 24:954958, 960, 962. 10.1271/bbb.62.1271

Nolan T, Hands RE, and Bustin SA. 2006. Quantification of mRNA using real-time RTPCR. Nat Protoc 1:1559-1582. 10.1038/nprot.2006.236

Pfaffl MW, Tichopad A, Prgomet C, and Neuvians TP. 2004. Determination of stable housekeeping genes, differentially regulated target genes and sample integrity: BestKeeper--Excel-based tool using pair-wise correlations. Biotechnol Lett 26:509-515. 10.1023/B:BILE.0000019559.84305.47

Remans T, Keunen E, Bex GJ, Smeets K, Vangronsveld J, and Cuypers A. 2014. Reliable gene expression analysis by reverse transcription-quantitative PCR: reporting and minimizing the uncertainty in data accuracy. Plant Cell 26:3829-3837. 10.1105/tpc.114.130641

Stürzenbaum SR, and Kille P. 2001. Control genes in quantitative molecular biological techniques: the variability of invariance. Comparative Biochemistry and Physiology Part B: Biochemistry and Molecular Biology 130:281-289. 10.1016/s1096-4959(01)00440-7

Stamova BS, Apperson M, Walker WL, Tian Y, Xu H, Adamczy P, Zhan X, Liu DZ, Ander BP, Liao IH, Gregg JP, Turner RJ, Jickling G, Lit L, and Sharp FR. 2009. Identification and validation of suitable endogenous reference genes for gene expression studies in human peripheral blood. BMC Med Genomics 2:49. 10.1186/1755-8794-2-49

Suzuki T, Honda Y, and Mukasa Y. 2005. Effects of UV-B radiation, cold and desiccation stress on rutin concentration and rutin glucosidase activity in tartary buckwheat (Fagopyrum tataricum) leaves. Plant Science 168:1303-1307. 10.1016/j.plantsci.2005.01.007

Terzi V, Morcia C, Spini M, Tudisco R, Cutrignelli MI, Infascelli F, Stanca AM, and Faccioli P. 2010. Identification and validation of reference genes for gene expression studies in water buffalo. Animal 4:853-860. 10.1017/S1751731110000042

Tian C, Jiang Q, Wang F, Wang GL, Xu ZS, and Xiong AS. 2015. Selection of suitable reference genes for QPCR normalization under abiotic stresses and hormone stimuli in carrot leaves. PLoS One 10:e0117569. 10.1371/journal.pone.0117569 
420

421

422

423

424

425

426

427

428

429

430

431

432

433

434

435

436

437

438

439

440

441

442

443

444

445

446

447

448

449

450

451

452

453

454

455

456
Tong Z, Gao Z, Wang F, Zhou J, and Zhang Z. 2009. Selection of reliable reference genes for gene expression studies in peach using real-time PCR. BMC Mol Biol 10:71. 10.1186/1471-2199-10-71

Vandesompele J, De Preter K, Pattyn F, Poppe B, Van Roy N, De Paepe A, and Speleman F. 2002. Accurate normalization of real-time quantitative RT-PCR data by geometric averaging of multiple internal control genes. Genome Biol 3:RESEARCH0034. 10.1186/gb-2002-3-7-research0034

Wan H, Zhao Z, Qian C, Sui Y, Malik AA, and Chen J. 2010. Selection of appropriate reference genes for gene expression studies by quantitative real-time polymerase chain reaction in cucumber. Anal Biochem 399:257-261. 10.1016/j.ab.2009.12.008

Wang H, Chen RF, Iwashita T, Shen RF, and Ma JF. 2015. Physiological characterization of aluminum tolerance and accumulation in tartary and wild buckwheat. New Phytol 205:273-279. 10.1111/nph.13011

Wu BY, Ye JR, Huang L, He LM, and Li DW. 2017. Validation of reference genes for RTqPCR analysis in Burkholderia pyrrocinia JK-SH007. J Microbiol Methods 132:9598. 10.1016/j.mimet.2016.10.004

Wu ZJ, Tian C, Jiang Q, Li XH, and Zhuang J. 2016. Selection of suitable reference genes for qRT-PCR normalization during leaf development and hormonal stimuli in tea plant (Camellia sinensis). Sci Rep 6:19748. 10.1038/srep19748

Yao H, Li C, Zhao H, Zhao J, Chen H, Bu T, Anhu W, and Wu Q. 2017. Deep sequencing of the transcriptome reveals distinct flavonoid metabolism features of black tartary buckwheat (Fagopyrum tataricum Garetn.). Prog Biophys Mol Biol 124:49-60. 10.1016/j.pbiomolbio.2016.11.003

Yuan T, Fujioka S, Takatsuto S, Matsumoto S, Gou XP, He K, Russell SD, and Li J. 2007. $B E N 1$, a gene encoding a dihydroflavonol 4-reductase (DFR)-like protein, regulates the levels of brassinosteroids in Arabidopsis thaliana. The Plant Journal 51:220-233. 10.1111/j.1365-313X.2007.03129.X

Zhang L, Li X, Ma B, Gao Q, Du H, Han Y, Li Y, Cao Y, Qi M, Zhu Y, Lu H, Ma M, Liu L, Zhou J, Nan C, Qin Y, Wang J, Cui L, Liu H, Liang C, and Qiao Z. 2017. The Tartary Buckwheat Genome Provides Insights into Rutin Biosynthesis and Abiotic Stress Tolerance. Mol Plant 10:1224-1237. 10.1016/j.molp.2017.08.013

Zhu H, Wang H, Zhu Y, Zou J, Zhao FJ, and Huang CF. 2015. Genome-wide transcriptomic and phylogenetic analyses reveal distinct aluminum-tolerance mechanisms in the aluminum-accumulating species buckwheat (Fagopyrum tataricum). BMC Plant Biol 15:16. 10.1186/s12870-014-0395-z 


\section{Figure 1}

$\mathrm{Cq}$ values of seven candidate reference genes across all experimental sets in tartary buckwheat.

A line across the box depicts the median. The box indicates the $25 \%$ and $75 \%$ percentiles. Whiskers represent the maximum and minimum values, and asterisks indicate extremes.

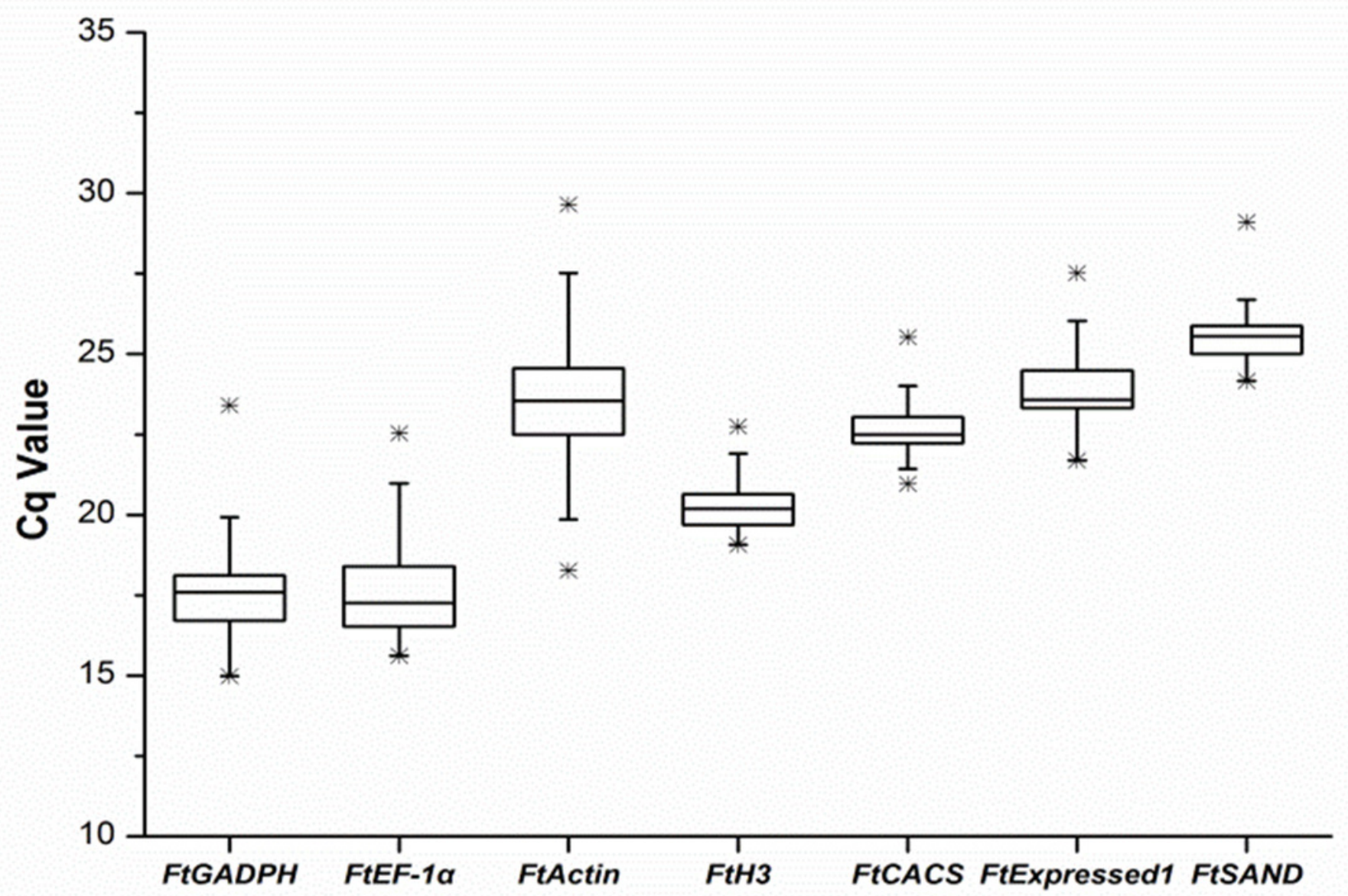


Figure 2

Optimal number of reference genes required for accurate normalization entile.

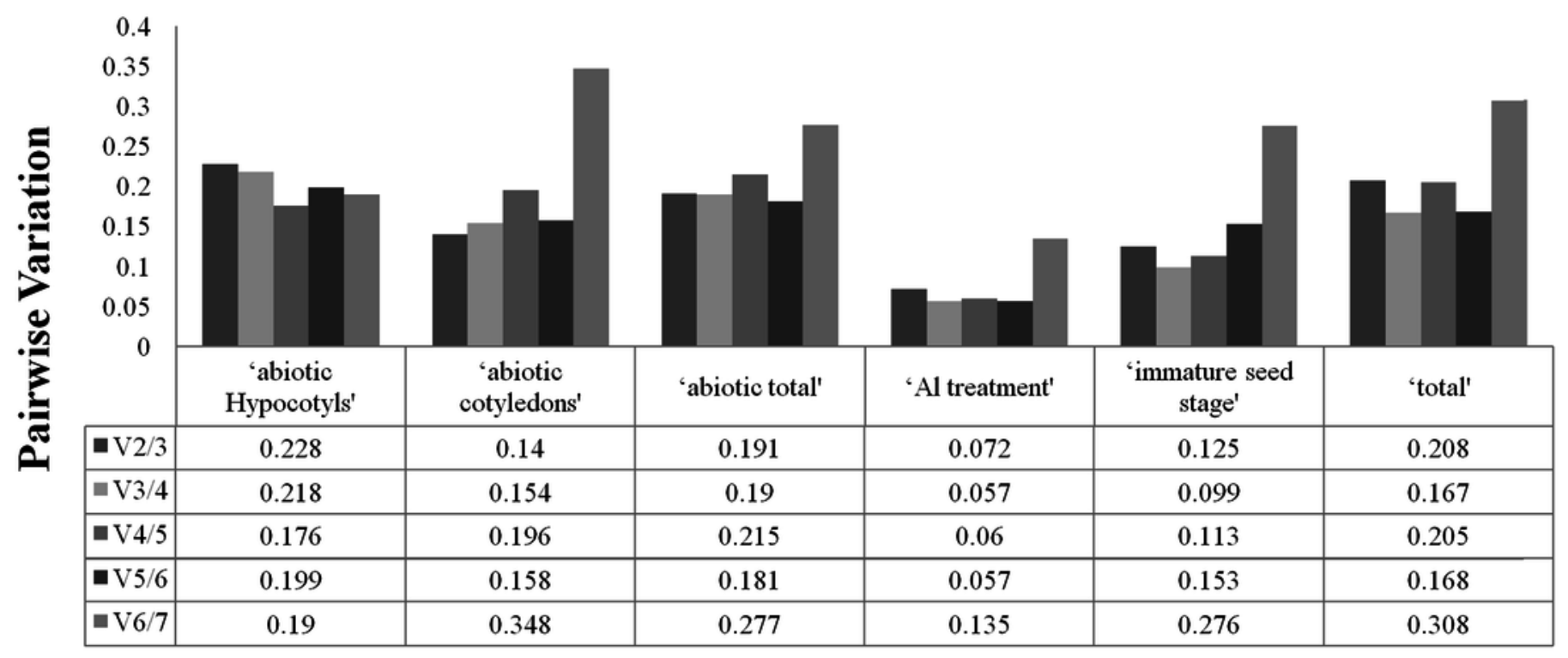


Figure 3

Relative quantification of FtSTAR2 gene expression under Al treatment in tartary buckwheat.

Error bars represent standard deviation of the mean, data shown are means \pm SD $(n=3)$.

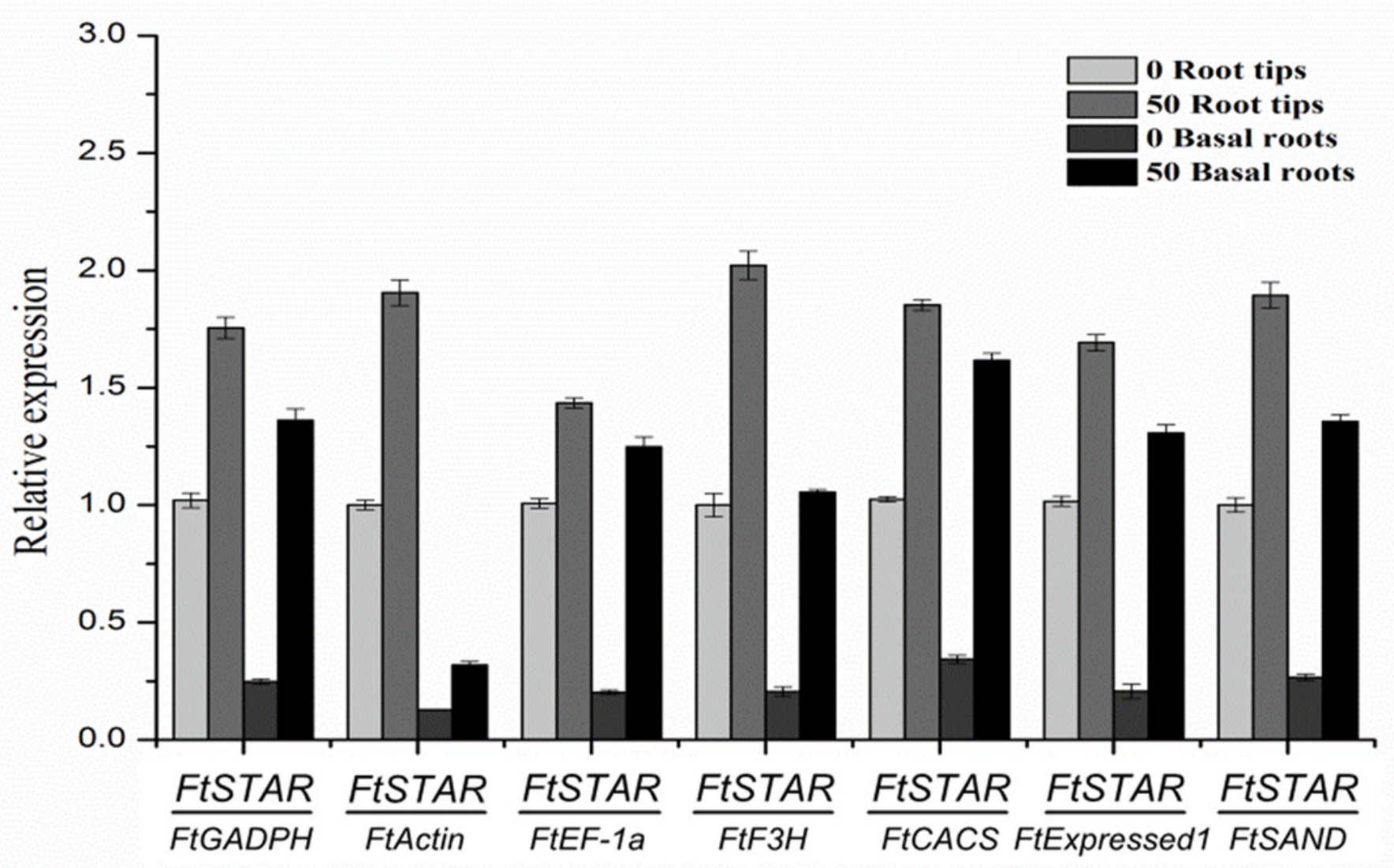


Figure 4

Relative quantification of FtDFR gene expression under UV treatment in tartary buckwheat hypocotyls.

Error bars represent standard deviation of the mean, data shown are means $\pm S D(n=3)$.

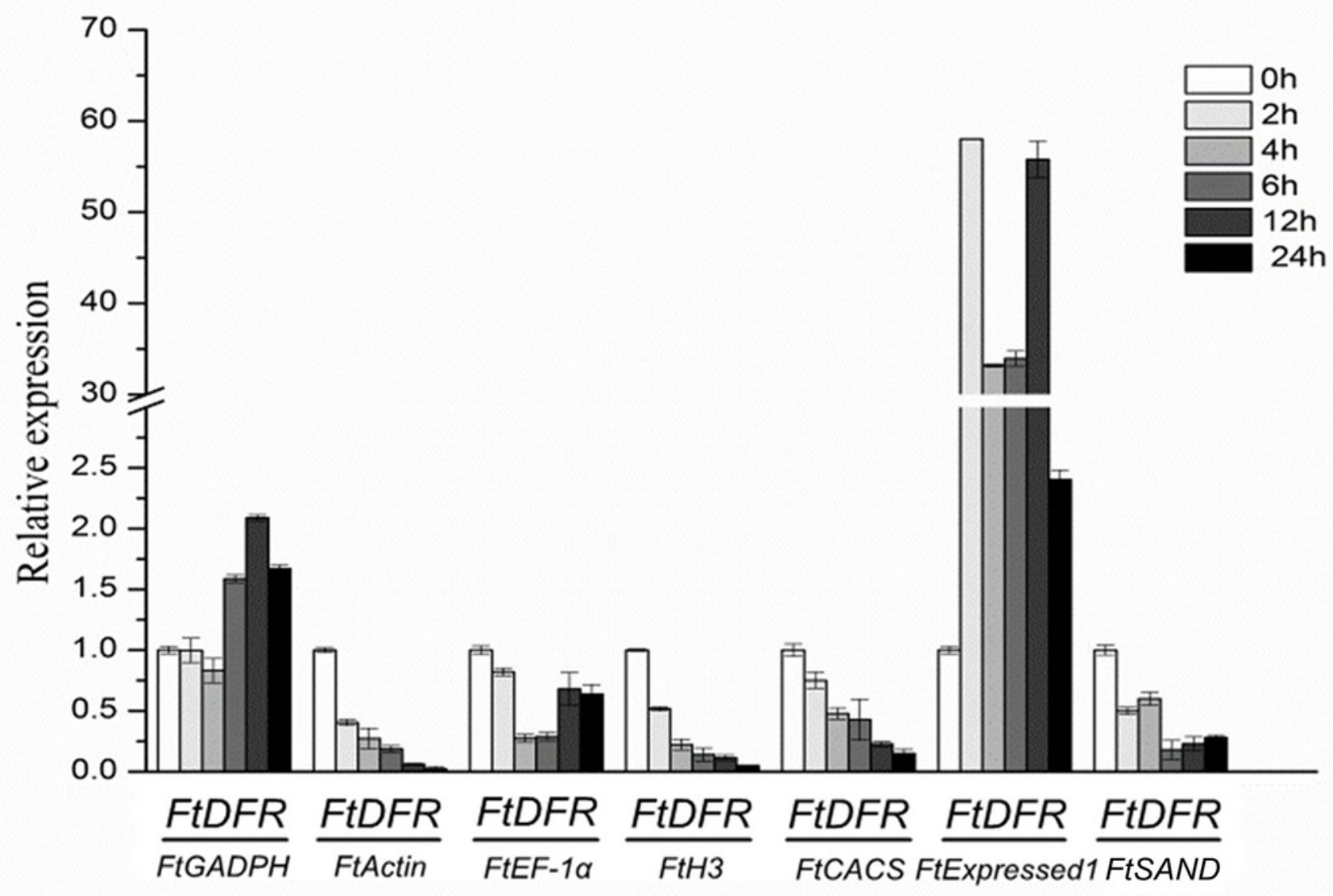




\section{Table 1 (on next page)}

Genes, primers and different features derived from qRT-PCR analysis 
1 Table 1 Genes, primers and different features derived from qRT-PCR analysis

\begin{tabular}{|c|c|c|c|c|c|}
\hline Gene symbol & Gene name & $\begin{array}{l}\text { Amplification } \\
\text { length (bp) }\end{array}$ & Primer sequences & $\mathrm{E} \%$ & $\mathrm{TM}^{\circ} \mathrm{C}$ \\
\hline FtSAND & SAND family protein gene & 79 & $\begin{array}{l}\text { GACCCCCTTGCAGACAAAGCATTGGCA } \\
\text { TCTCGTTCTCAACGTCTTTTACCCACTGG }\end{array}$ & 98.9 & 81 \\
\hline FtCACS & $\begin{array}{c}\text { Clathrin adapter complex subunit } \\
\text { family protein }\end{array}$ & 125 & $\begin{array}{l}\text { AAGACAGTCAGTTTCGTGCCACCTGA } \\
\text { TCCATGCGTGTTCTACCCAACTCCTT }\end{array}$ & 90.3 & 82.5 \\
\hline FtExpressed 1 & $\begin{array}{c}\text { Expressed protein of unknown } \\
\text { function }\end{array}$ & 127 & $\begin{array}{l}\text { AGGCCAGTTCCTGCTGAATGTAATGC } \\
\text { TAGCCTGATCCAAACAAGCCTGGCAA }\end{array}$ & 90.9 & 83 \\
\hline $\mathrm{FtH}_{3}$ & Histones 3 & 158 & $\begin{array}{l}\text { GAAATTCGCAAGTACCAGAAGAG } \\
\text { CCAACAAGGTATGCCTCAGC }\end{array}$ & 109.3 & 85 \\
\hline FtGAPDH & $\begin{array}{c}\text { Glyceradehyde-3-phosphate } \\
\text { debydrogenase gene }\end{array}$ & 155 & $\begin{array}{c}\text { TGGAGCTGCTAAGGCTGTCG } \\
\text { TGATAGCACTCTTGATGTCCTCGTA }\end{array}$ & 91.9 & 83 \\
\hline FtActin & Actin 2 genes & 118 & $\begin{array}{c}\text { GGAAGTATAGCGTCTGGATTGGC } \\
\text { CACTTGCGGTGAACGATTGC }\end{array}$ & 93.1 & 82.5 \\
\hline$F t E F-1 \alpha$ & Elongation factor- $1 \alpha$ gene & 108 & $\begin{array}{l}\text { GCTGCTGAGATGAACAAGAGGTC } \\
\text { CTCAAACTTCCACAACGCGAT }\end{array}$ & 91.2 & 82.5 \\
\hline
\end{tabular}




\section{Table 2 (on next page)}

The stability of the expression of seven candidate reference genes in six groups as analysed by geNorm, NormFinder, BestKeeper 
1 Table 2 The stability of the expression of seven candidate reference genes in six groups as 2 analysed by geNorm, NormFinder, BestKeeper

\begin{tabular}{|c|c|c|c|c|c|c|c|c|}
\hline \multirow{2}{*}{ Group } & \multirow{2}{*}{ Rank } & \multicolumn{2}{|c|}{ geNorm } & \multicolumn{2}{|c|}{ NormFinder } & \multicolumn{3}{|c|}{ BestKeeper } \\
\hline & & Gene & Stability & Gene & Stability & Gene & SD & $\mathrm{CV}$ \\
\hline \multirow{7}{*}{$\begin{array}{l}\text { Abiotic } \\
\text { Cotyledo }\end{array}$} & 1 & FtCACS & 0.33 & $\mathrm{FtH}_{3}$ & 0.058 & FtSAND & 0.33 & 1.32 \\
\hline & 2 & $\mathrm{FtH}_{3}$ & 0.33 & FtCACS & 0.080 & FtCACS & 0.31 & 1.47 \\
\hline & 3 & $F t S A N D$ & 0.41 & $F t S A N D$ & 0.129 & $\mathrm{FtH}_{3}$ & 0.33 & 1.64 \\
\hline & 4 & FtExpressed 1 & 0.54 & FtExpressed 1 & 0.280 & FtExpressed 1 & 0.59 & 2.48 \\
\hline & 5 & $F t E F-1 \alpha$ & 0.73 & $F t E F-1 \alpha$ & 0.683 & $F t E F-1 \alpha$ & 1.04 & 5.70 \\
\hline & 6 & FtGAPDH & 0.84 & FtGAPDH & 0.751 & FtGAPDH & 1.07 & 6.26 \\
\hline & 7 & FtActin & 1.31 & FtActin & 1.678 & FtActin & 1.46 & 6.36 \\
\hline \multirow{7}{*}{$\begin{array}{l}\text { Abiotic } \\
\text { Hypocot }\end{array}$} & 1 & $\mathrm{FtH}_{3}$ & 0.65 & FtSAND & 0.416 & $\mathrm{FtH}_{3}$ & 0.51 & 2.47 \\
\hline & 2 & FtSAND & 0.65 & FtCACS & 0.437 & FtSAND & 0.69 & 2.68 \\
\hline & 3 & FtCACS & 0.72 & $\mathrm{FtH}_{3}$ & 0.449 & FtActin & 0.79 & 3.38 \\
\hline & 4 & FtExpressed 1 & 0.84 & $F t E F-1 \alpha$ & 0.469 & FtExpressed 1 & 0.81 & 3.34 \\
\hline & 5 & $F t E F-1 \alpha$ & 0.91 & FtExpressed 1 & 0.475 & FtCACS & 0.86 & 3.75 \\
\hline & 6 & FtGAPDH & 1.05 & FtGAPDH & 0.811 & $F t E F-1 \alpha$ & 0.92 & 5.32 \\
\hline & 7 & FtActin & 1.17 & FtActin & 0.895 & FtGAPDH & 1.35 & 7.59 \\
\hline \multirow{7}{*}{$\begin{array}{c}\text { Abiotic } \\
\text { total }\end{array}$} & 1 & $\mathrm{FtH}_{3}$ & 0.55 & $F t C A C S$ & 0.304 & $\mathrm{FtH}_{3}$ & 0.52 & 2.55 \\
\hline & 2 & FtSAND & 0.55 & FtSAND & 0.327 & FtCACS & 0.61 & 2.70 \\
\hline & 3 & $F t C A C S$ & 0.61 & $\mathrm{FtH}_{3}$ & 0.349 & $F t S A N D$ & 0.63 & 2.46 \\
\hline & 4 & FtExpressed 1 & 0.72 & FtExpressed 1 & 0.380 & FtExpressed 1 & 0.73 & 3.02 \\
\hline & 5 & $F t E F-1 \alpha$ & 0.89 & $F t E F-1 \alpha$ & 0.694 & $F t E F-1 \alpha$ & 1.02 & 5.71 \\
\hline & 6 & FtGAPDH & 1.01 & FtGAPDH & 0.782 & FtActin & 1.17 & 5.07 \\
\hline & 7 & FtActin & 1.30 & FtActin & 1.329 & FtGAPDH & 1.20 & 6.89 \\
\hline \multirow{7}{*}{$\begin{array}{c}\mathrm{Al} \\
\text { treatmen }\end{array}$} & 1 & $F t E F-1 \alpha$ & 0.16 & FtSAND & 0.091 & FtExpressed 1 & 0.17 & 0.73 \\
\hline & 2 & FtExpressed 1 & 0.16 & FtExpressed 1 & 0.100 & $F t E F-1 \alpha$ & 0.25 & 1.55 \\
\hline & 3 & FtSAND & 0.21 & $\mathrm{FtH}_{3}$ & 0.105 & $\mathrm{FtH}_{3}$ & 0.31 & 1.56 \\
\hline & 4 & FtGAPDH & 0.23 & $F t E F-1 \alpha$ & 0.106 & FtGAPDH & 0.34 & 1.88 \\
\hline & 5 & $\mathrm{FtH}_{3}$ & 0.27 & FtGAPDH & 0.156 & FtCACS & 0.35 & 1.57 \\
\hline & 6 & FtCACS & 0.30 & FtCACS & 0.282 & FtSAND & 0.37 & 1.43 \\
\hline & 7 & FtActin & 0.49 & FtActin & 0.652 & FtActin & 1.05 & 4.16 \\
\hline Immatur & 1 & FtExpressed 1 & 0.16 & FtSAND & 0.097 & FtExpressed 1 & 0.50 & 2.00 \\
\hline
\end{tabular}




\begin{tabular}{|c|c|c|c|c|c|c|c|c|}
\hline e seed & 2 & FtCACS & 0.16 & FtGAPDH & 0.097 & FtCACS & 0.57 & 2.43 \\
\hline \multirow[t]{5}{*}{ stage } & 3 & FtSAND & 0.30 & $\mathrm{FtH}_{3}$ & 0.167 & FtSAND & 0.82 & 3.03 \\
\hline & 4 & FtGAPDH & 0.36 & FtCACS & 0.292 & $F t E F-1 \alpha$ & 0.83 & 4.32 \\
\hline & 5 & $\mathrm{FtH}_{3}$ & 0.45 & FtExpressed 1 & 0.422 & FtGAPDH & 0.86 & 4.51 \\
\hline & 6 & $F t E F-1 \alpha$ & 0.62 & $F t E F-1 \alpha$ & 0.675 & $\mathrm{FtH}_{3}$ & 1.11 & 5.29 \\
\hline & 7 & FtActin & 1.01 & FtActin & 1.354 & FtActin & 2.16 & 9.96 \\
\hline \multirow{7}{*}{ Total } & 1 & $\mathrm{FtH}_{3}$ & 0.60 & FtCACS & 0.245 & FtCACS & 0.53 & 2.33 \\
\hline & 2 & FtCACS & 0.60 & FtSAND & 0.304 & $\mathrm{FtH}_{3}$ & 0.54 & 2.69 \\
\hline & 3 & FtSAND & 0.66 & $\mathrm{FtH}_{3}$ & 0.313 & FtSAND & 0.57 & 2.24 \\
\hline & 4 & FtExpressed 1 & 0.71 & FtExpressed 1 & 0.377 & FtExpressed 1 & 0.68 & 2.85 \\
\hline & 5 & FtGAPDH & 0.87 & FtGAPDH & 0.650 & FtGAPDH & 0.97 & 5.53 \\
\hline & 6 & $F t E F-1 \alpha$ & 0.97 & $F t E F-1 \alpha$ & 0.780 & $F t E F-1 \alpha$ & 1.03 & 5.91 \\
\hline & 7 & FtActin & 1.33 & FtActin & 1.480 & FtActin & 1.36 & 5.70 \\
\hline
\end{tabular}

3 